\title{
EL ESTRUCTURALISMO EN LA ENSEÑANZA DEL IDIOMA INGLÉS
}

\section{THE STRUCTURALISM IN THE TEACHING OF THE ENGLISH LANGUAGE}

Mgs. Hugo Hernán Romero Rojas ${ }^{1}$

Mgs. Marcela Patricia Gonzalez Robalino

Mgs. Jacqueline Guadalupe Armijos Monar ${ }^{3}$

Universidad Nacional de Chimborazo , Riobamba, Ecuador

\section{RESUMEN}

El presente trabajo parte brevemente del concepto de paradigma, la adquisición de la lengua materna versus el inglés como

$\overline{1}{ }^{*}$ Magíster en lingüística aplicada al aprendizaje del Inglés. Diplomado Superior en Metodologías para el aprendizaje del idioma Inglés. Profesor Titular Universidad Nacional de Chimborazo, Riobamba, Ecuador hromero@unach.edu.ec 0981007230 https://orcid.org/00000001-8335-0599

$2 \quad$ Magíster en lingüística aplicada al aprendizaje del Inglés. Diplomado Superior en Metodologías para el aprendizaje del idioma Inglés, Profesora Titular, Universidad Nacional de Chimborazo, Riobamba, Ecuador marcepago76@gmail.com 0995718741 https://orcid. org/0000-0002-4772-6090

$3 \quad$ Magíster en lingüística aplicada al aprendizaje del Inglés, Diplomado Superior en Metodologías para el aprendizaje del idioma Inglés, Profesora Titular, Universidad Nacional de Chimborazo, Riobamba, Ecuador igarmijosmonar@gmail.com 0958842089. https://orcid. org/0000-0001-7580-2292 segunda lengua; posteriormente, se plantea el estructuralismo en la enseñanza del idioma inglés por medio de una propuesta de análisis sintáctico (sujeto, predicadoy sus modificadores), tomando en cuenta las combinaciones de las categorías gramaticales en oraciones simples con verbos transitivos. Finalmente se ejemplifica un ejercicio de sustitución.

PALABRAS CLAVE: estructuralismo, funciones gramaticales, oraciones simples.

This article briefly starts from the concept of paradigm, the acquisition of the mother tongue versus English as a second language, later, the structuralism in the teaching of the English language is contemplated through a proposal of syntactic analysis (subject, predicate and its 
modifiers ), taking into account the combinations of grammatical categories in simple sentences with transitive verbs. Finally, a substitution exercise is exemplified.

\section{SUMMARY}

KEYWORDS: Structuralism, grammatical functions, simple sentences.

A inicios del siglo XVII el estadounidense Thomas Kuhn publica su trabajo cumbre $L a$ Estructura de las Revoluciones Científicas. Por medio de esta obra aparece el concepto de paradigma. Para Kuhn un paradigma es un conjunto de realizaciones científicas universalmente reconocidas que durante cierto tiempo proporcionan modelos de problemas y soluciones a una comunidad científica. (Raffone, 2015)

Paradigma, es una guía del quehacer para la exploración de la comprensión... modelo, o ejemplo que se realiza para encontrar contestación a las dificultades que el desconocimiento propone (mayéutica socrática, la deducción aristotélica, la lógica formal con sus relaciones circulares entre premisas y conclusión y sus modos de formular nuevas propuestas; la inducción originadora del empirismo, el cartesianismo que dio lugar al deductivismo...); una forma de comprender, una ruta destacada de creación de conocimiento... (Montero, 2005)

Paradigma científico es una constelación de alcances, términos, valores, técnicas, etc. que tiene una comunidad científica en común y que son aplicados por esa comunidad para la definición de problemas y soluciones legítimas. Paradigma Social sería una constelación de términos, valores, percepciones y prácticas que tienen en común una comunidad y que forma una determinada vista, percepción de la realidad que es el substrato de la manera como esta sociedad se auto-organiza. (Capra, 1996)
El paradigma también se lo puede considerar como el conjunto de los supuestos teóricos generales, las leyes, las técnicas de aplicación adoptados por los miembros de una comunidad científica para estudiar fenómenos y problemas.

Este breve análisis es imprescindible antes de partir con la temática. El inglés hoy en día se lo considera como la clave del éxito que permite abrir las puertas del conocimiento. La primera lengua o lengua materna tiene prevalencia en los planes de estudio tanto en las horas como en el desarrollo de la misma en comparación con el inglés, este no se lo practica o se lo desarrolla en nuestro medio en donde el español impera, en esta abismal diferencia yace el valor de la enseñanza y la adquisición del idioma extranjero por parte de los educandos.

La lengua materna es aquella que entra en contacto directo con el niño a través de sus progenitores, familiares y amigos, lo que no sucede con la segunda lengua que se encuentra fuera del entorno sociolingüístico, su exposición al inglés se lo realiza en la escuela y dependiendo el número de horas semanales si es una escuela pública o privada. Si una lengua no oficial es utilizada para la enseñanza es considerada como lengua extranjera, la cual se convirtió en un instrumento de uso común entre las diferentes clases sociales, permitiendo de esta forma tener acceso a redes y entornos virtuales y a bases de datos científicos. ( Krashen 1981)

El inglés se lo adquiere en nuestro medio en una institución educativa, no es común o habitual la comunicación en segunda lengua, más bien, la primera lengua es la utilizada a diario en todo tipo de eventos de tipo laboral, educativo, cultural, etc. En el Ecuador entendemos que cuando nos comunicamos en inglés la estamos utilizando como lengua extranjera porque la lengua materna en la que interactuamos es el español. La enseñanza del inglés en el Ecuador se ha institucionalizado en los centro educativos 
públicos y privados gracias a los acuerdos ministeriales, en 1992 se firmó un convenio entre el British Council y el Ministerio de Educación, en el cual se implementa una reforma curricular en la enseñanza del inglés en la educación secundaria. (British council, 2015)

Cuando se da un movimiento migratorio las personas están obligadas a comunicarse en un nuevo idioma, algunos compatriotas que viajaron a los Estados Unidos experimentaron un aprendizaje obkigatorio y necesario para poder subsistir y convivir con sus congéneres en el desarrollo de sus actividades. (Hernández, 2014).

Las teorías lingüísticas en el transcurso del tiempo han influenciado en los cambios de paradigma en la enseñanza del idioma inglés. Hasta el año de 1970 la lingüística estructural predominó, en este período el currículo de enseñanza del inglés se regía en lo estructural. Ferdinand de Saussure fue el padre del Estructuralismo, quien contradictoriamente no plasmó en ningún escrito su sapiencia, fueron sus discípulos Bally y Sechehaye recuperaron sus enseñanzas en el libro Curso de Lingüística General.

Las escuelas estructuralistas fueron tres: la de Praga con Troubeskoy y Jacobson; la de Copenhague Louis Hjelmslev; y la de Norteamérica con Bloomfield y Harris. El predomino del estructuralismo ha sido evidente en los diferentes métodos de enseñanza del idioma inglés, así tenemos los siguientes:

Grammar Translation Method (Método de Traducción Gramatical).

Direct Method (Método Directo)

Método Audio-Lingual

Total Physical Response (Método de Respuesta Física Total).
Silent Method (Método Silencioso)

Suggestopedia method (Método de Sugestopedia) (Madany-Saa, 2013)

En el Ecuador, durante la clases de inglés las oraciones eran presentadas y esquematizadas de acuerdo a las funciones gramaticales o sintácticas,

Las funciones sintácticas son terminales de relaciones contraídas linealmente en determinado contexto (oración, construcción). Las funciones se llenan con palabras o construcciones... (Kovacci, 1990)

asítenemosnúcleodelsujetoysusmodificadores: modificador directo $(\mathrm{md})$, modificador indirecto (mi); el núcleo del predicado y sus modificadores; objeto directo (OD), objeto indirecto (OI) y los complementos circunstanciales (CC).

El objeto directo (OD) es un modificador monovalente de voz activa, se duplica con los pronombres objetivos (lo, la, los, las y le,les)...

El objeto indirecto $(\mathrm{OI})$ se representa o se duplica con el pronombre personal objetivo de tercera persona en sus formas le (se),les, y por conmutación con ellas, las objetivas de primera y segunda persona. (Kovacci, 1990)

El complemento circunstancial no puede ser sujeto en voz pasiva y no puede ser conmutable por un pronombre objetivo. El agente puede ser sujeto en voz activa, no puede ser conmutable por un pronombre objetivo. (Barrenechea, 1984) 


\section{PROPUESTA DE ANÁLISIS SINTÁCTICO}

Dada la oración

The beautiful girl in black sings a song.

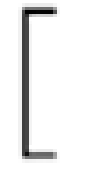<smiles>CCCC</smiles>

Aplico los siguientes pasos:

1. Utilización de los corchetes para la delimitación de la oración simple.

2. Identificación del verbo flexionado: sings, este verbo desempeña la función de núcleo de la oración.

3. Separación de la oración en sujeto (S) y predicado $(\mathrm{P})$.

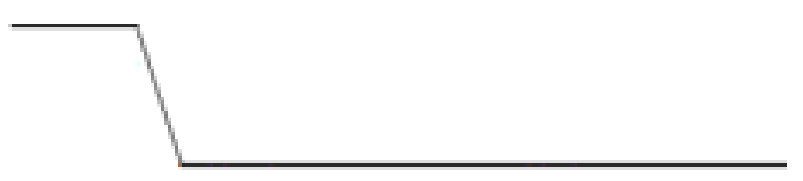

4. El sujeto es simple, la categoría gramatical es el sustantivo que desempeña esta función es girl.

5. El núcleo del sujeto girl está acompañado de dos modificadores The y beautiful, la categoría gramatical de estas palabras es adjetivo, un adjetivo puede desempeñar la función gramatical de modificador directo (md). In black es un modificador indirecto porque inicia con una preposición in.

6. La naturaleza del núcleo del predicado es verbal sings es un verbo flexionado, por su clasificación sintáctica es un verbo transitivo, entonces, de acuerdo a su construcción es exocéntrica, está presente el enfrentamiento entre sujeto y predicado.

7. A song desempeña la función gramatical de objeto directo, es posible aplicar las dos operaciones formales de la pronominalización y la pasivización para su análisis.

8. Pronominalización: The beautiful girl sings it. It reemplaza a: a song

9. Pasivización: A song is sang by the beautiful girl. El objeto directo pasa a ser sujeto y el sujeto the beautiful girl pasa a ser agente. Otra forma de comprobación es utilizando la palabra this (para el singular), these (para el plural), en este ejemplo This is sang by the beautiful girl. This reemplaza a: a song.

Si a la oración The beautiful girl in black sings a song incrementa to Mary, tenemos:

10. Identificar el objeto indirecto to Mary. El procedimiento a seguir es la comprobación que se logra por medio de la pronominalización, reemplazamos to Mary con un pronombre personal objetivo de la tercera persona her, acompañado de la palabra to. Es importante aclarar que en español sería el pronombre objetivo le que sirve tanto para el género masculino como femenino. La oración sería The beautiful girl in black sings a song to her, her reemplaza a Mary.

Ahora bien, si a la oración le incrementamos in the mornings, tenemos:

The beautiful girl in black sings a song to Mary in the mornings.

11. Identificamos el complemento circunstancial que corresponde a tiempo mornings y que empieza con la preposición in. 
[

En estos once pasos se plantea la propuesta para analizar las oraciones simples con verbos transitivos.

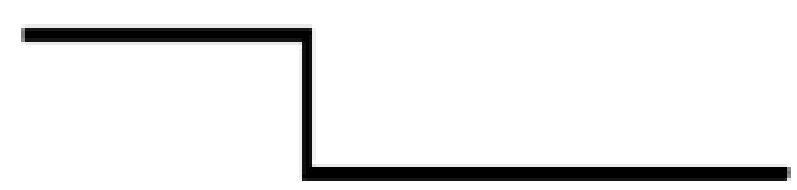

Sujeto

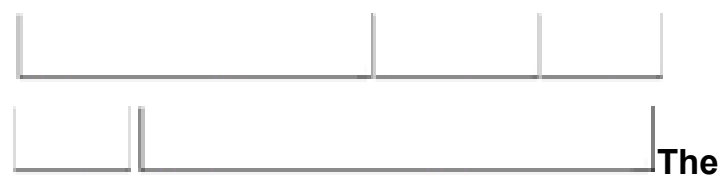

beautiful girl in black sings a song to Mary in the mornings. MD $\mathrm{N} \quad \mathrm{MD} \quad \mathrm{MI}$ $\mathrm{N}$ OD OI C C Predicado (Oración simple)

Las clases de las palabras pueden ser las siguientes: sustantivos, adjetivos, verbos, verboides (infinitivos, gerundios y participios), preposiciones, conjunciones y relacionantes. Todas estas categorías pueden ser descritas según tres criterios el semántico, morfológico y sintáctico. Estas clases llamadas también categorías gramaticales pueden ser reemplazadas en su misma categoría, de esta forma en las clases de inglés el profesor aplicaba los ejercicios de sustitución en inglés denominado substitution drills.

The beautiful girl in black sings a song to Mary in the mornings.

1. Con adjetivos.

Bad, green. The bad girl in green

Sad, yellow. The sad girl in yellow
Tall, red. The tall girl in red

2. Con sustantivos

Boy. The bad boy in green

Student. The bad student in yellow

Person. The bad person in red

3. Con verbos.

Writes. The bad boy in green writes a song to Mary in the mornings.

Sends. The sad student in yellow sends a song to Mary in the mornings.

Reads. The bad person in red reads a song to Mary in the mornings.

4. Con nouns (OD)

A book. The bad boy in green writes a book to Mary in the mornings.

A tale. The sad student in yellow sends a tale to Mary in the mornings.

A story. The bad person in red reads a story to Mary in the mornings.

5. Sustantivos (OI)

To Michael. The bad boy in green writes a book to Michael in the mornings.

To Jennifer. The sad student in yellow sends a tale to Jennifer in the mornings.

To Rachel. The bad person in red reads a story to Rachel in the mornings.

6. Con adverbios

At noon. The bad boy in green writes a book to Michael at noon.

In the afternoons. The sad student in yellow sends a tale to Jennifer in the afternoons. 
In the evenings. The bad person in red reads a story to Rachel in the evenings.

\section{CONCLUSIONES}

Constituye una nueva propuesta de análisis que contrasta el inglés con el español.

Se puede analizar cualquier oración simple que tenga un verbo transitivo.

Constituye una base para los principiantes en el análisis sintáctico de las oraciones.

Por medio de la sustitución de palabras de la misma categoría gramatical se pueden obtener tantas oraciones como el número de verbos transitivos que se conozca, es decir el manejo de un vocabulario básico.

Permite comprender las funciones sintácticas tanto del idioma español como del inglés.

Su utilización y práctica es muy sencilla para los aprendices del idioma inglés.

La terminología utilizada corresponde al estructuralismo, esto es importante destacar porque en la teoría lingüística del generativismo se utilizan otros términos.

\section{BIBLIOGRAFÍA}

Barrenechea, A. (1984). Estudios de gramática estructural. Buenos Aires: Editorial Paidós.

British Council. (2015). Recuperado el 16 de 03 de 2018, de https://www. teachingenglish.org.uk/sites/teacheng/ files/English\%20in\%20Ecuador.pdf

Capra, F. (1996). La trama de la vida: una nueva perspectiva de los sistemas vivos. Barcelona: Anagrama.

Hernández, E. (2014). Enseñanza del idioma inglés. Recuperado el 28 de Febrero de 2019, de https://eprints.ucm.es/29610/1/
T35913.pdf

Kovacci, O. (1990). El comentario gramatical . Madrid: Grafur, S.A.

Krashen, S. (1981). Second language acquisition and second language learning. Oxford: Pergamon Press.

Madany-Saa, M. (11 de Noviembre de 2013). Second Language Aacquisition and Approches to Language. ( $\mathrm{H}$. Romero, Entrevistador)

Montero, M. (1 de Abril de 2005). Scielo. Recuperado el 8 de Marzo de 2019, de http://www.scielo. org.ve/scielo.php?pid=S1316$00872005000100002 \&$ script $=$ sci arttext\&tlng=pt

Raffone, C. (17 de 09 de 2015). Scrib. Recuperado el 15 de 06 de 2019, de https://www. scribd.com/document/281481109/KuhnParadigmas-Ciencia 